\title{
Characterization of the Shielding Effects on the Frequency-Dependent Effective Dielectric Constant of a Waveguide-Shielded Microstrip Using the Finite-Difference Time-Domain Method
}

\author{
Lin-Kun Wu, Member, IEEE, and Yeung-Chung Chang
}

\begin{abstract}
The dispersion behavior of waveguide-shielded microstrip line is investigated using the finite-difference timedomain method. The result is a frequency-dependent effective dielectric constant. Structures having centered strip are examined to determine the effects of a top cover alone and the effects of two symmetrically placed sidewalls. Structures with offcentered strip are used to investigate the effects of a single sidewall alone and the combined effects of a single sidewall plus the top wall. Differences between the effects of a single sidewall alone and those of the two symmetrically placed sidewalls are identified. In addition, new results on the combined effects of the top wall plus one sidewall, which are important when considering the placement of the outermost elements of a packaged circuit, are also discussed.
\end{abstract}

\section{INTRODUCTION}

$I^{N}$ $\mathrm{N}$ many practical applications a microstrip circuit entails a metallic enclosure, which provides hermetic sealing, mechanical strength, electromagnetic shielding, connector mounting, and ease of handling of the module [1]. The presence of a housing places an upper limit on the single-mode (i.e., quasi-TEM) operating frequency. In addition, within this frequency range, both the characteristic impedance and the effective dielectric constant are decreased owing to the physical proximity of the housing, and this should be taken into account during initial design and analysis of the circuit [2].

Conventionally, the shielding effects are considered as separate perturbations from one of the sidewalls and from the top cover alone [1]-[4]. More recently, the effects of waveguide housing on the dispersive properties of a microstrip with the strip centered between the two sidewalls were examined briefly while investigating the $90^{\circ}$ microstrip bend [5]. In another development, the

Manuscript received January 9, 1991; revised May 6, 1991. This work was supported by the National Science Council of the Republic of China under Grant NSC 79-0404-E009-05.

The authors are with the Institute of Communication Engineering, National Chiao Tung University, 1001 Ta Hsueh Road, Hsinchu, Taiwan 30050.

IEEE Log Number 9102322.

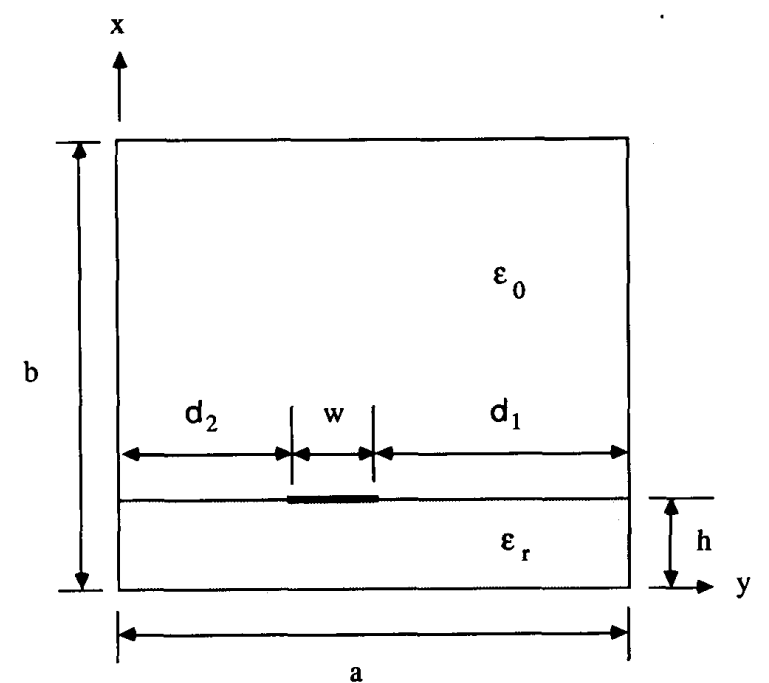

Fig. 1. Cross-sectional view of the waveguide-shielded uniform microstrip line with arbitrary strip position with respect to the two sidewalls. (Note that $w=h=0.635 \mathrm{~mm}$ and $\epsilon_{r}=9.7$ are used throughout the study.)

effects of a shielding cavity were analyzed using the method of moments [6]. These studies fail to distinguish between effects of a single sidewall and those of two symmetrically placed sidewalls. This issue and the combined effects of a single sidewall plus the top wall, which are important when considering the placement of the outermost circuit element (with respect to both the top lid and the nearby sidewall), are investigated here.

In this research, the finite-difference time-domain (FDTD) method developed in [7] and [8] for analyzing open microstrip structures is first modified to take into account the presence of the conducting top lid and sidewalls. It is then employed to analyze various waveguideshielded microstrip structures with arbitrary strip position as shown in Fig. 1. Both centered and off-centered strip 
(with respect to the two sidewalls) geometries are used to determine the effects of the individual wall and to determine their combined effects.

The FDTD technique is chosen for its ability to determine accurately the broad-band characteristics of a wide range of microstrip structures by simulating the propagation of a Gaussian pulse through each individual structure [5], [7], [8]. In the following, a brief account of the FDTD method and of the procedures used to determine the frequency-dependent effective dielectric constant will be presented, followed by a discussion of the results obtained for various waveguide-shielded microstrip structures.

\section{Summary of the Analysis Technioue}

Fig. 1 shows the microstrip structure used in this study. The strip conductor is assumed to be an infinitesimally thin perfect conductor. It is placed on top of a lossless isotropic dielectric substrate having a thickness $h$. The uniform microstrip line is then placed into a metallic waveguide housing of size $a \times b$. The spacings between the strip and each of the two sidewalls, $d_{1}$ and $d_{2}$, are arbitrary. The entire structure extends uniformly into infinity along both the $\pm z$ directions.

To determine the shielding effects on microstrip dispersion, propagation of a Gaussian pulse along the shielded microstrip line is analyzed using the modified version of the FDTD method given in [7] and [8]. In this study, the pulse width is chosen to cover the frequency range from dc to just below the cutoff frequency of the first higher order waveguide mode. For simplicity, the latter is approximated by that of the structure shown in Fig. 1 but with the strip conductor removed [6].

The FDTD technique proceeds by first segmenting the shielded microstrip structure into a three-dimensional mesh composed of a number of unit cells. Spatial and temporal discretizations of the electric and magnetic field components then follow. The positioning of the electric and magnetic field components about a unit cell in rectangular coordinates is shown in Fig. 2. Temporal sampling instants for the electric field components and the magnetic field components are also interleaved, with a half time step separation between them. In this research, the spatial sampling interval is chosen to be one-eighth the substrate thickness, which has been found to yield converged and accurate results. To satisfy the Courant stability criterion [9] and to ease the implementation of the superabsorbing boundary condition [10], the time step is chosen such that the dc component of the pulse will propagate across one space cell in the $z$ direction in five time steps.

A proper value of the permittivity is assigned to each electric field component of the lattice except for the field sampling points at the air-dielectric interface; here the average permittivity of the two media is used to satisfy the field continuity condition at the air-dielectric interface [8]. Appropriate tangential electric field components and

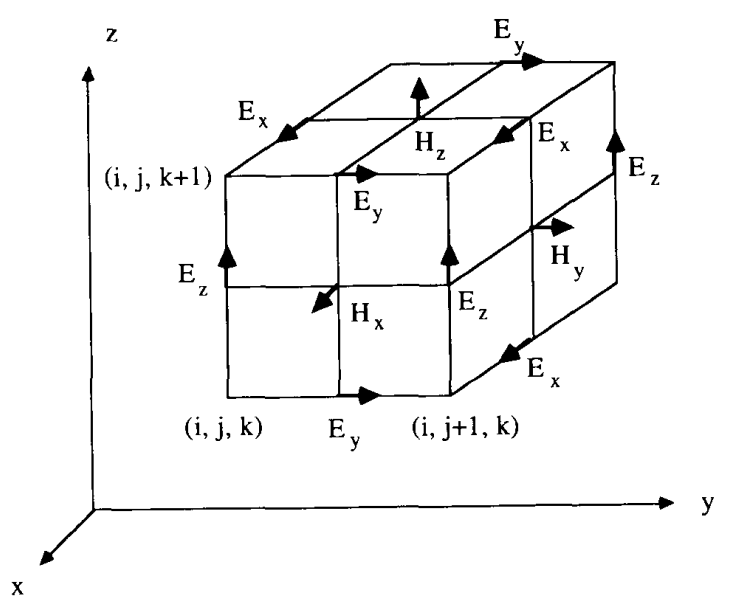

Fig. 2. Unit cell in rectangular coordinates.

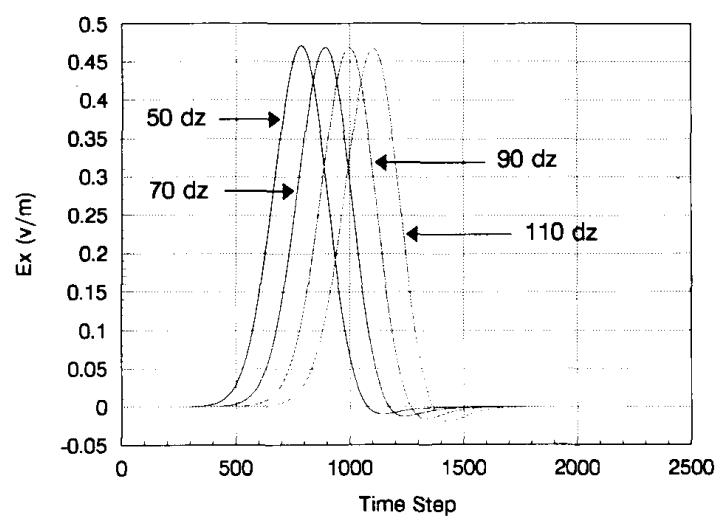

Fig. 3. Time waveforms of $E_{x}$ taken half a cell below the center of the strip conductor and at $z=50,70,90$, and 110 cells away from the excitation plane. The strip is symmetrically placed between sidewalls and the size of the housing is $10 h \times 7 h$.

the normal magnetic field components are placed on the surfaces of the waveguide walls and the strip conductor; on the strip, only $E_{z}$ is allowed to be placed at the edge of the strip. The boundary conditions are enforced by setting these field quantities to zero at all times. In addition, the superabsorbing boundary condition [10] is utilized to terminate the FDTD problem space in the direction of wave propagation.

The initial condition is such that a null field is present in the entire FDTD lattice except at the plane of excitation in which the Gaussian pulse has just arrived. It is further assumed that the excitation source is present only in, and distributed uniformly over, the portion of the substrate underneath the strip conductor [8]. Following this, a simple leapfrog time-stepping algorithm [11] is employed to simulate wave propagation through the FDTD lattice.

Fig. 3 shows the space-time variations of $E_{x}$ observed at several locations (all half a cell below the center of the 
strip) in the $z$ direction for the centered strip case with a housing size of $10 h \times 7 h$. It is seen that, owing to the dispersion in the microstrip structure, distortion in the pulse increases as it propagates farther down the line. The problem space is truncated at $z=120 d z$ in this case. To check the performance of the superabsorbing boundary condition, the same structure is analyzed again with the problem space truncated at $200 \mathrm{dz}$. Time waveforms of $E_{x}$ observed at $z=90 \mathrm{dz}$ in the two cases are compared. It is found that differences between the two waveforms attain a maximum value of about $0.0002 \mathrm{~V} / \mathrm{m}$, or about $0.05 \%$ of the peak value of $E_{x}$ observed at $z=90 \mathrm{dz}$ shown in Fig. 3. Excellent performance of the superabsorbing boundary condition is thus expected.

To determine $\epsilon_{r \text { eff }}(\omega)$ for the shielded microstrip structure considered, $E_{x}(t)$ at two different locations separated by a distance $L$ in the $z$ direction are Fourier transformed, with the resultant $z$-dependent spectra denoted by $E_{x}(\omega, z=0)$ and $E_{x}(\omega, z=L)$, respectively. Since only a propagating quasi-TEM mode is present in the frequency range considered, and both the strip conductor and the dielectric substrate are assumed to be lossless, the resultant spectra are related by the frequency-dependent phase constant $\beta(\omega)$ as follows:

$$
E_{x}(\omega, z=L)=E_{x}(\omega, z=0) e^{-j \beta(\omega) L}
$$

where

$$
\beta(\omega)=\omega\left[\mu_{0} \epsilon_{0} \epsilon_{r \text { eff }}(\omega)\right]^{1 / 2} .
$$

These equations are used to determine $\epsilon_{r \text { eff }}(\omega)$ from the FDTD simulated results.

\section{Results And Discussions}

The results to be presented in this section are all obtained for a strip having a unity strip-width-to-substrate-height ratio (i.e., $w / h=1$ ) placed on top of a $0.635-\mathrm{mm}$-thick alumina substrate with $\epsilon_{r}=9.7$. Centered strip geometry is used when examining the effects of the two symmetrically placed sidewalls. The structural symmetry consideration indicates that a magnetic wall is present at the plane of symmetry (i.e., $y=a / 2$ ), which thus reduces the problem space by half. On the other hand, an off-centered strip position is used for investigating the effects of a single sidewall. In this case, one of the sidewalls is placed far from the strip conductor such that it will not interact with the fringing field lines originating from the strip. The spacing between the second sidewall and the strip is then varied within a range where interaction between the two is expected. The additional top-cover effects are studied by adjusting the height of the housing simultaneously.

\section{A. Centered-Strip Structures}

Before the effects of a single sidewall and the combined effects of top wall plus one sidewall are investigated

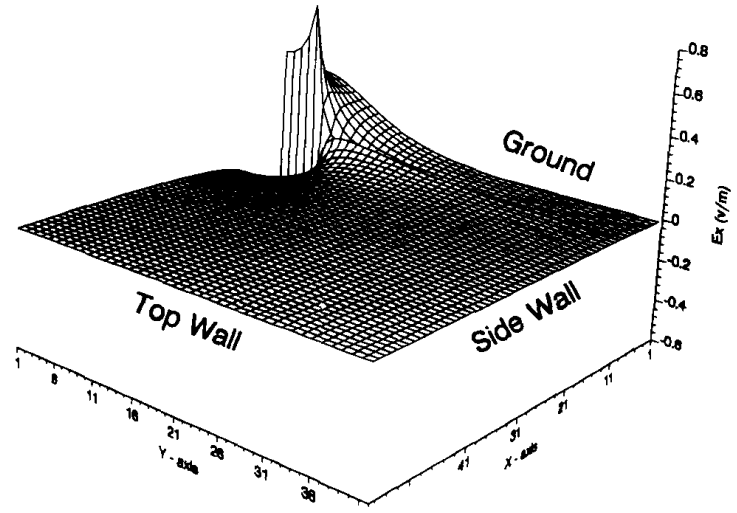

Fig. 4. Plot of $E_{x}(0 \leqslant x \leqslant b, a / 2 \leqslant y \leqslant a)$ for a centered-strip structure having a housing size of $10 h \times 7 h$ when pulse reaches the maximum at a half cell below the strip.

by analyzing the entire structure, an extensive numerical experiment is undertaken with the strip conductor centered between the two sidewalls (i.e., $d_{1}=d_{2}$ ). Results obtained from this set of experiments characterize the effects of two symmetrically placed sidewalls and/or top cover, and from these one can identify the minimum sidewall and top wall spacings required so that their interactions with the fringing fields can be ignored.

Ranges of $a / h$ from 4 to 12 and of $b / h$ from 2 to 8 are used in this set of experiments. It is found that, for all $b / h$ ratios considered, $\epsilon_{r \text { eff }}(\omega)$ remains essentially unchanged when $a / h$ exceeds about 10 (i.e., $d_{1} / h=d_{2} / h$ $\geqslant 4.5$ ). Fig. 4 shows the spatial field distribution of $E_{x}(x, y)$ taken for a housing size of $10 h \times 7 h$ when $E_{x}$ half a cell below the center of the strip conductor reaches its maximum value. It is found that fields are concentrated mainly in the immediate surroundings of the strip conductor; no field line is found to terminate at the top wall. However, small amounts of $E_{x}$ do exist along the air-dielectric interface between the edge of the strip and the sidewall. Apparently, this latter phenomenon does not perturb to any significant degree the field distribution of $E_{x}$ half a cell below the center of the strip, and $\epsilon_{r \text { eff }}(\omega)$ remains essentially unchanged when $a / h$ exceeds about 10.

The effects of the top cover alone can then be determined, and these are summarized in Fig. 5 for $a / h=10$ and $b / h$ from 2 to 7 . The figure shows clearly that the effects of the top cover alone may be ignored in calculating $\epsilon_{r \text { eff }}$ when $b / h \geqslant 7$ because, as shown in Fig. 4, no field line can reach the top wall. On the other hand, the effects of the top cover become quite significant when $b / h$ is less than 4 . This is more obvious at low frequencies as the wave-guiding capability of the strip is weaker and more fringing fields are present in the air region to interact with the top wall. Finally, by extrapolating the curve down to dc for the case of $b / h=2$ (i.e., the strip is centered between the top and the bottom wall), a theoretical $\epsilon_{r \text { eff }}$ value of 5.35 (i.e., $\left.\left(\epsilon_{r}+1\right) / 2\right)$ is obtained. 


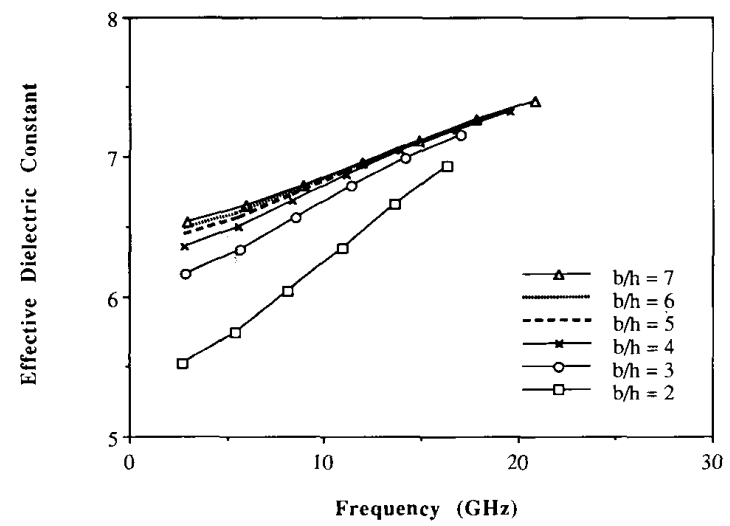

Fig. 5. The effects of lowering the top cover for fixed $a / h=10$ (i.e., $d_{1} / h=d_{2} / h=4.5$ ).

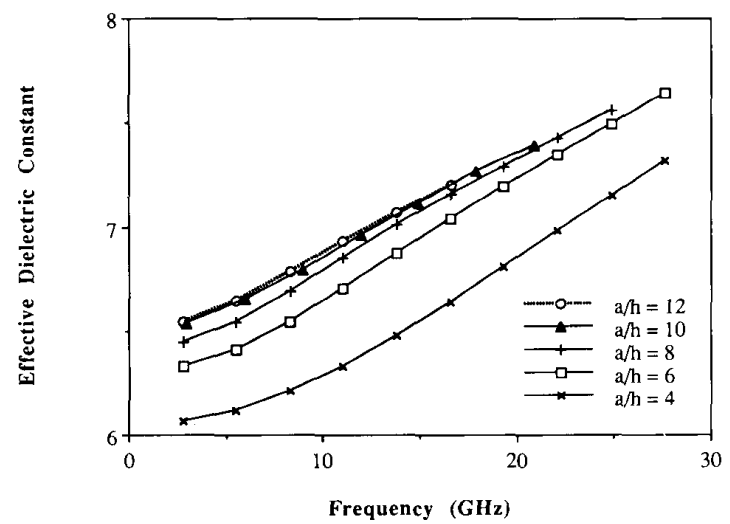

Fig. 6. The effects of closing in the two sidewalls with the normalized sidewall-to-strip spacing $d_{1} / h=d_{2} / h=(a / h-w / h) / 2$. The topcover height is fixed at $b / h=7$.

Based on the above observations, the effects of two symmetrically placed sidewalls (i.e., $d_{1} / h=d_{2} / h$ ) can then be examined from the $\epsilon_{r \text { eff }}$ data shown in Fig. 6 , which are obtained by fixing $b / h=7$ while varying $a / h$ from 4 to 12 . The figure shows that data obtained for $a / h=10$ and 12 are almost identical. On the other hand, reductions in $\epsilon_{r \text { eff }}$ can be detected when $a / h$ is less than 10 (i.e., $d_{1} / h=d_{2} / h \leqslant 4.5$ ), which decreases rapidly for $a / h \leqslant 8$ (i.e., $d_{1} / h=d_{2} / h \leqslant 3.5$ ).

These observations indicate that for the centered-strip structure considered the effects of waveguide housing may be ignored when a housing size of $10 h \times 7 h$ is used. To verify the accuracy of the FDTD method, results obtained for a housing size of $10 h \times 7 h$ are compared with the open-microstrip data calculated from the closedform formulas of Kobayashi [12] and Hammerstad and Jensen [13] in Fig. 7. As can be seen, our data are in good agreement with those calculated using Kobayashi's formula over the entire frequency range considered for the shielded microstrip case. However, although Hammerstad

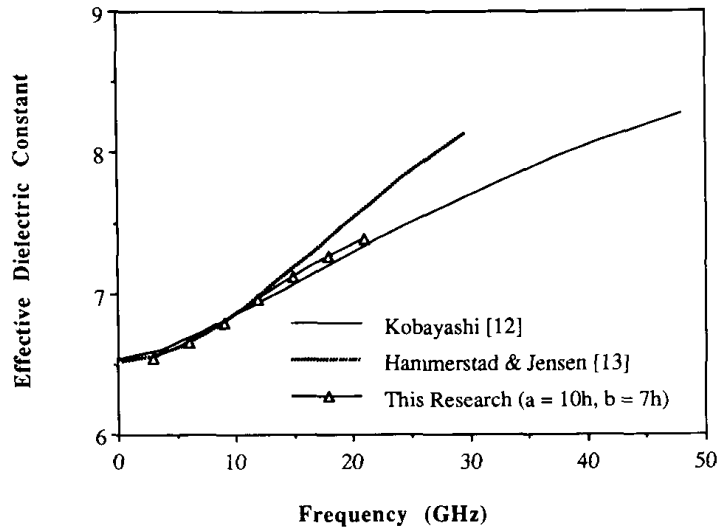

Fig. 7. Comparison of $\epsilon_{r \text { eff }}(\omega)$ obtained from this research for a centered-strip structure having a housing size of $10 h \times 7 h$ with the openmicrostrip data calculated using closed-form formulas given in [12] and $[13]$.

and Jensen's formula results in a comparable $\epsilon_{r \text { eff }}$ at lower frequencies, it yields much higher values of $\epsilon_{r \text { eff }}$ at higher frequencies. Since the $\epsilon_{r \text { eff }}(\omega)$ data used in the development of the dispersion formula in [12] are calculated from a rigorous spectral-domain approach with carefully selected basis and weighting functions [14], they are considered to be more accurate. As such, this comparison confirms the accuracy achievable by the FDTD method.

\section{B. Off-Centered Strip Structure}

In view of the results observed in the numerical experiments performed for the centered-strip structures, the effects of a single sidewall, with and without any additional effects of the top cover, may be characterized by using an off-centered strip geometry with $d_{1} / h=4.5$ (fixed), $d_{2} / h<4.5$, and $b / h \leqslant 7$. Results thus obtained are compared with those determined for the centered-strip case with $a / h=10$ and $b / h=7$, for which both the top cover and the two sidewalls may be ignored.

As our first example, results obtained with $d_{2} / h$ varying from 3.5 to 0.5 for $b / h=7$ are shown in Fig. 8. It is noted that, since the top wall and one of the two sidewalls are far enough away so that they do not interact with the strip conductor, $\epsilon_{r \text { eff }}(\omega)$ obtained for $d_{2} / h<4.5$ can be regarded as the odd-mode $\epsilon_{r \text { eff }}(\omega)$ for unshielded parallel-coupled microstrip lines spaced $2 d_{2} / h$ apart. To better illustrate the effects of a single approaching sidewall, data obtained for the centered-strip case with $d_{2} / h=4.5$ are also plotted in Fig. 8. It is found that the effects of a single sidewall are insignificant when $d_{2} / h=3.5$ but become quite significant for smaller $d_{2} / h$. Furthermore, an examination of the data shown in Figs. 6 and 8 indicates that, for $d_{2} / h=3.5$ or 2.5 , the amount of decrease in $\epsilon_{r \text { eff }}(\omega)$ almost doubled when two symmetrically placed sidewalls are present compared with the corresponding single-sidewall cases. However, the reduc- 


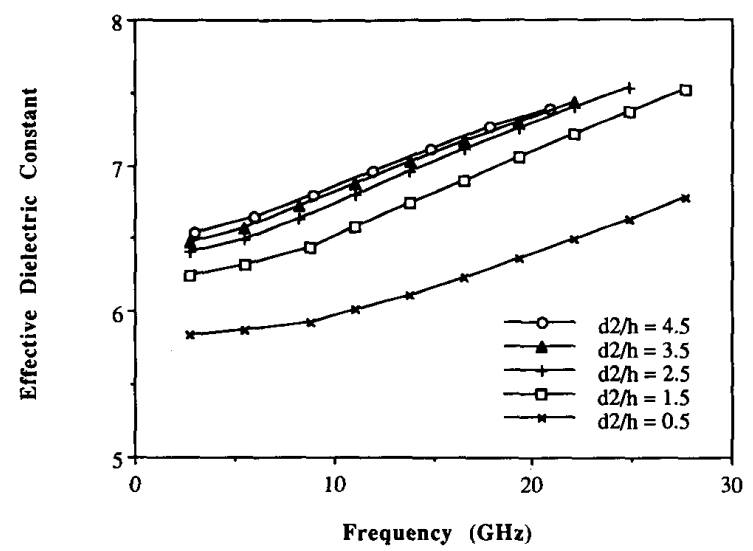

Fig. 8. The effects of a single approaching sidewall for the case of off-centered strip geometry with fixed $b / h=7$ and $d_{1} / h=4.5$.

tion factor is less than 2 when $d_{2} / h=1.5$. This suggests that the effects of a single sidewall and those of two symmetrically placed sidewalls should be addressed separately.

As the top cover height is lowered to $b / h=6$ and 5 , results almost identical to those shown in Fig. 8 are obtained for each of the four $d_{2} / h$ values considered, which indicates that the effects of the approaching sidewall prevail when $b / h \geqslant 5$. However, by comparing the results obtained for $b / h=4$ shown in Fig. 9(a) with the corresponding data shown in Fig. 8 , it is found that the approaching top wall results in a further reduction in $\epsilon_{r \text { eff }}$. The amount of reduction is found to be larger at lower frequencies and for larger values of $d_{2} / h$ as more fields are present in the air-filled region of the structure to interact with the approaching top lid. Finally, parts (b) and (c) of Fig. 9 show that, for each of the four $d_{2} / h$ values considered, much smaller values of $\epsilon_{r \text { eff }}$ are observed as the top cover height is further lowered to $b / h=3$ and 2 .

\section{Remarks on the Computational Aspects of the FDTD Method}

It is generally recognized that the FDTD method, while being able to solve a wide range of problems with a minimum mathematical preprocessing effort, is highly demanding computationally. Take this study, for instance; 140 cells (with $\delta=h / 8$ ) were used along the $z$ axis for the data presented here. In all cases, data converging to within $1 \%$ were obtained when $E_{x}(\omega)$ taken for $z \geqslant 110 \delta$ were used in (1). For the case of a centered strip with a large housing size of $10 h \times 7 h$, the total mesh dimensions in the $x, y$, and $z$ directions and the total time steps simulated were $40 \times 56 \times 140 \times 2500$. The CPU time for this particular problem was about $6 \mathrm{~h}$ on an IBM RS $6000 / 320$ workstation (which is rated at 27 MIPS/7.5 MFLOPS). In another test, a CPU time of $20 \mathrm{~min}$ was measured when the housing size was reduced to $4 h \times 2 h$,

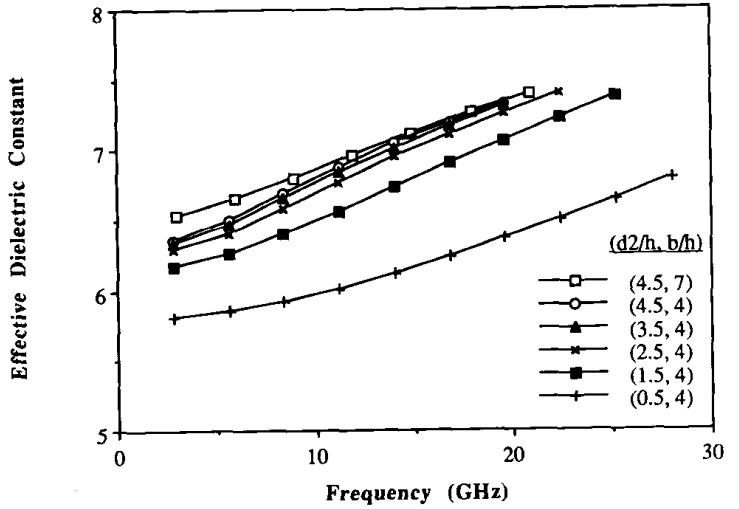

(a)

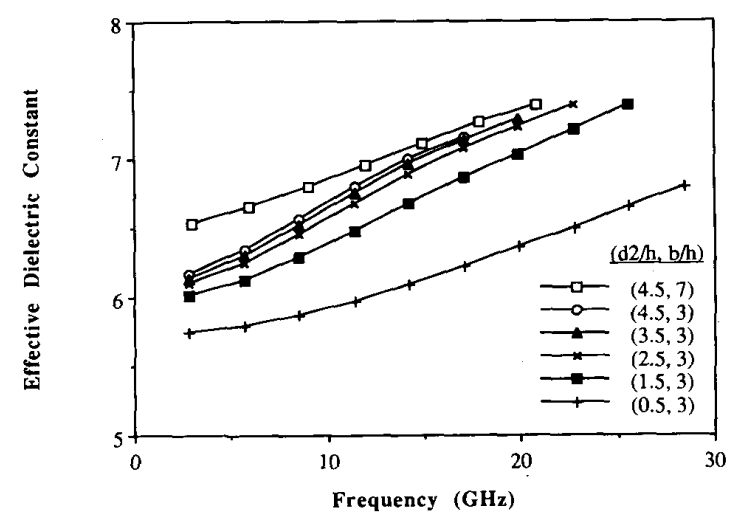

(b)

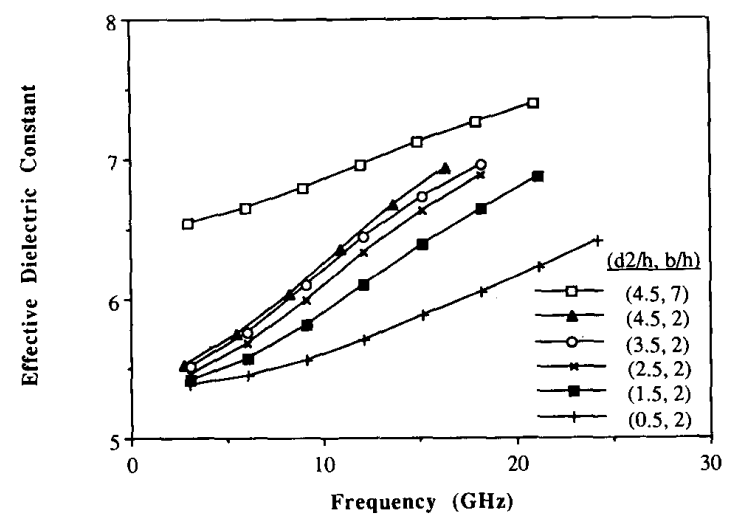

(c)

Fig. 9. The combined effects of a single sidewall plus the top wall, with $d_{1} / h=4.5$ fixed, for (a) $b / h=4$, (b) $b / h=3$, and (c) $b / h=2$. Data obtained for the centered strip case with housing size of $10 \mathrm{~h} \times 7 \mathrm{~h}$ are also shown in each case for comparison.

for which the mesh dimensions were $16 \times 16 \times 140$ in space and 2000 in time.

As evidenced by the field plot shown in Fig. 4, rapid variation in the field distribution is expected only near the strip conductor, use of a 3-D variable mesh scheme for improving its computational efficiency may be justified. In 
this regard, an extension of the 2-D local mesh refinement scheme described in [15] deserves further attention.

\section{Conclusions}

The FDTD method has been employed to investigate the effects of shielding on the dispersive properties of a waveguide-shielded microstrip line on alumina substrate. Both centered and off-centered strip geometries have been examined to identify the effects of (1) the top cover alone, (2) two symmetrically placed sidewalls, (3) a single sidewall, and (4) the top wall plus one sidewall. For the centered-strip cases, it is found that the top cover may be ignored in calculating $\epsilon_{r \text { eff }}(\omega)$ when $b / h \geqslant 7$. On the other hand, the sidewall effects are insignificant when the spacing between the sidewall and the edge of the strip exceeds about 4.5 times the substrate thickness. For the off-centered strip cases, it is observed that the shielding effects are mainly due to the approaching sidewall when $d_{2} / h<4.5$ and $b / h>4$. On the other hand, the combined effects of a single sidewall plus the top cover should be considered when $d_{2} / h<4.5$ and $b / h<4$. Finally, compared with the effects of a single approaching sidewall with $d_{2} / h=3.5$ or 2.5 , reductions in $\epsilon_{r \text { eff }}(\omega)$ almost double when two sidewalls with $d_{1} / h=d_{2} / h$ are present.

\section{REFERENCES}

[1] T. C. Edwards, Foundations for Microstrip Circuit Design. New York: Wiley, 1981

[2] S. March, "Microstrip packaging: Watch the last step," Microwales, pp. 83-94, Dec. 1981

[3] R. K. Hoffmann, Handbook of Microwate Integrated Circuits Norwood, MA: Artech House, 1987.

[4] E. H. Fooks and R. A. Zakarevicius, Microwace Engineering Using Microstrip Circuits. Englewood Cliffs, NJ: Prentice-Hall, 1990.

[5] J. Moore and H. Ling "Characterization of a $90^{\circ}$ microstrip bend with arbitrary miter via the time-domain finite difference method." IEEE Trans. Microwace Theory Tech., vol. 38, pp. 405-410, Apr. 1990

6] L. P. Dunleavy and P. B. Katehi, "Shielding effects in microstrip discontinuities," IEEE Trans. Microwace Theory Tech., vol, 36, pp. 1767-1774, Dec. 1988.

[7] X. Zhang, J. Fang, K. K. Mei, and Y. Liu, "Calculations of the dispersive characteristics of microstrips by the time-domain finite difference method," IEEE Trans. Microware Theory Tech., vol. 36. pp. $263-267$. Feb. 1988

[8] X. Zhang and K. K. Mei, "Time-domain finite difference approach to the calculation of the frequency-dependent characteristics of microstrip discontinuities," IEEE Trans. Microwace Theory Tech.. vol. 36, pp. 1775-1787, Dec. 1988.
[9] A. Taflove and M. E. Brodwin, "Numerical solution of steady-state electromagnetic scattering problems using the time-dependent Maxwell's equations," IEEE Trans. Microwate Theory Tech., vol. MTT-23, pp. 623-630, Aug. 1975.

[10] J. Fang and K. K. Mei, "A super-absorbing boundary algorithm for solving electromagnetic problems by time-domain finite-difference method," in IEEE AP-S Int. Symp. Dig., June 1988, pp. 472-475.

[11] K. S. Yee, "Numerical solution of initial boundary value problems involving Maxwell's equations in isotropic media," IEEE Trans. Antennas Propagat., vol. AP-14, pp. 302-307, May 1966.

[12] M. Kobayashi, "A dispersion formula satisfying recent require ments in microstrip CAD," IEEE Trans. Microwace Theory Tech. vol. 36, pp. 1246-1250, Aug. 1988.

[13] E. Hammerstad and O. Jensen, "Accurate models for microstrip computer-aided design," in IEEE MTT-S Int. Microwace Symp. Dig.. 1980 , pp. $407-409$

[14] M Kobayashi and F Ando, "Dispersion characteristics of open microstrip lines," IEEE Trans. Microwave Theory Tech., vol. MTT 35, pp. 101-105, Feb. 1987.

[15] I. S. Kim and W. J. R. Hoefer, "A local mesh refinement algorithm for the time domain-finite difference method using Maxwell's cur equations," IEEE Trans. Microwale Theory Tech., vol. 38, pp. $812-815$, June 1990

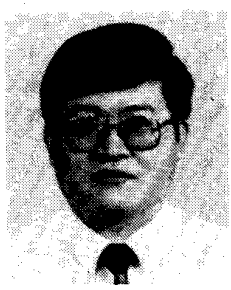

Lin-Kun Wu (S'81-M'85) was born in Hsinchu, Taiwan, Republic of China, on November 1 , 1958. He graduated from the Taipei Institute of Technology in 1978 and received the M.S. and $\mathrm{Ph}$.D. degrees in electrical engineering from the University of Kansas, Lawrence, in 1982 and 1985 , respectively.

From November 1985 to December 1987, he was a Postdoctoral Research Associate at the Center for Research, Inc., of the University of Kansas working on microwave remote sensing and computational electromagnetics. Since February 1988, he has been an Associate Professor at the Institute of Communication Engineering, National Chiao Tung University, Hsinchu, Taiwan. His current research interests involve the development of the finite-difference time-domain method and the method of moments for the characterization of microstrip and waveguide discontinuities and for the analysis of electromagnetic scattering problems. He is also interested in radar systems and radar remote sensing.

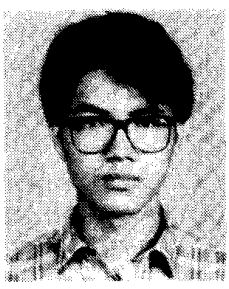

Yeung-Chung Chang was born in Sanchung, Taipei, Taiwan, Republic of China, on November 18,1966 . He received the B.S. degree in communication engineering in 1989 from National Chiao Tung University, Hsinchu, Taiwan, where he is currently working toward the M.S. degree. His B.S. and M.S. theses deal with the analysis of satellite antenna and the characterization of shielding effects on microstrip dispersion. His research interests include antennas, radar, and fiber optics. 